\title{
A METRIC ON HYPERSPACES DEFINED BY WHITNEY MAPS
}

\author{
WLODZIMIERZ J. CHARATONIK
}

ABSTRACT. For a given continuum $X$ a ncw metric on the hyperspace $2^{X}$ is defined, which is equivalent to the Hausdorff distance, but which has some other properties.

All spaces in this paper are assumed to be metric and all mappings are continuous. A continuum is a compact connected space. Given a continuum $X$ with a metric $d$, we define the Hausdorff distance $H$ between two nonempty closed subsets $A$ and $B$ by

$$
H(A, B)=\max \left\{\sup _{a \in A} \inf _{b \in B} d(a, b), \sup _{b \in B} \inf _{a \in A} d(a, b)\right\}
$$

(see [1, (0.4), p. 3]). The symbol $2^{X}$ denotes the hyperspace of all nonempty closed subsets of a continuum $X$ with the Vietoris topology (see [1, $(0.11)$, p. 9] for the definition) or, equivalently (see $[1,(0.13)$, p. 10]) with the topology determined by the Hausdorff distance.

A mapping $\mu: 2^{X} \rightarrow[0, \infty)$ is called a Whitney map (see $[1,(0.50)$, p. 24]) if it satisfies the conditions:

(1) for every $x \in X, \mu(\{x\})=0$; and

(2) for every $A, B \in 2^{X}$ with $A \subset B$ and $A \neq B, \mu(A)<\mu(B)$.

We consider special Whitney maps, namely ones satisfying an additional condition:

(3) for every $A, B \in 2^{X}$ with $A \subset B$ and for every $C \in 2^{X}$,

$$
\mu(B \cup C)-\mu(A \cup C) \leqslant \mu(B)-\mu(A) .
$$

Such mappings do exist for every continuum $X$ (see Proposition 1 below).

Given a sequence of sets $\left\{A_{n}\right\}_{n=1}^{\infty}$ we denote by Ls $A_{n}$ the upper limit of the sequence in the sense of $\left[1,(0.5)\right.$, p. 4], and by $\operatorname{Lim} A_{n}$ the limit of the sequence in the sense of $[1,(0.5)$, p. 4$]$ or, equivalently (see $[1,(0.7)$, p. 4$])$, in the sense of the Hausdorff distance.

In the present paper a new metric on the hyperspace of a continuum is defined, which is equivalent to the Hausdorff distance, but which has some other properties.

Received by the editors March 26, 1984 and, in revised form, July 9, 1984. 1980 Mathematics Subject Classification. Primary 54B20; Secondary 54E35, 54F15.

Key words and phrases. Hyperspace, continuum, Hausdorff distance, Whitney map. 
We start with

Proposition 1. For every continuum $X$ there are Whitney maps $\mu$ and $\mu^{\prime}$ such that $\mu$ satisfies, while $\mu^{\prime}$ does not satisfy, condition (3).

Really, the reader can verify that a Whitney map $\mu$ defined in $[1,(0.50 .2)$, p. 26] has property (3). On the other hand, let $x, y, z \in X$ be any distinct points and put $f(\{x\})=f(\{y\})=f(\{z\})=0, f(\{x, y\})=f(\{x, z\})=f(\{y, z\})=1$, and $f(\{x, y, z\})=3$. Then $f$ satisfies (1) and (2) for the space $\{x, y, z\}$ and therefore it can be extended to a Whitney map $\mu^{\prime}$ on $2^{X}$ (see [2, Corollary 3.4, p. 468] and observe that the assumption of connectedness of spaces is not used in the proof). However, putting $A=\{x\}, B=\{x, y\}$, and $C=\{z\}$, we can see that $f$ (and hence $\left.\mu^{\prime}\right)$ does not satisfy (3).

Definition 2. Let $X$ be a continuum and let $\mu$ be a Whitney map satisfying (3). Define, for every $P, Q \in 2^{X}$,

$$
D_{\mu}(P, Q)=\max \{\mu(P \cup Q)-\mu(P), \mu(P \cup Q)-\mu(Q)\} .
$$

Proposition 3. $D_{\mu}$ defined above is a metric on $2^{X}$.

Proof. The condition $D_{\mu}(P, Q)=0$ if and only if $P=Q$ is a consequence of (2); the symmetry of $D_{\mu}$ is obvious from the definition. We show the triangle condition. Let $P, Q, R \in 2^{X}$. We can assume without loss of generality that $\mu(P) \leqslant \mu(R)$. Then we have to show

$$
\begin{aligned}
\mu(P \cup Q)-\min \{\mu(P), \mu(Q)\}+\mu(Q \cup R)-\min \{\mu(Q), \mu(R)\} & \\
& \geqslant \mu(P \cup R)-\mu(P) .
\end{aligned}
$$

It is enough to show

$$
\mu(P \cup Q)-\mu(P)+\mu(Q \cup R)-\mu(Q)-\mu(P \cup R)+\mu(P) \geqslant 0,
$$

but using (3) for $A=Q, B=P \cup Q$, and $C=R$ we see that the left member of the inequality is greater than or equal to

$$
\mu(P \cup Q \cup R)-\mu(Q \cup R)+\mu(Q \cup R)-\mu(P \cup R)
$$

and, therefore, is nonnegative.

Proposition 4. For any Whitney map $\mu$ satisfying (3) the metric $D_{\mu}$ is equivalent to the Hausdorff distance $H$.

Proof. Let a set $A \in 2^{X}$ be given and assume a sequence $\left\{A_{n}\right\}_{n=1}^{\infty}$ tends to $A$ with respect to the Hausdorff distance, i.e., $H\left(A_{n}, A\right) \rightarrow 0$. Then $H\left(A_{n} \cup A, A\right) \rightarrow 0$, and by continuity of $\mu$ we have $\mu\left(A_{n} \cup A\right) \rightarrow \mu(A)$ and $\mu\left(A_{n}\right) \rightarrow \mu(A)$. Thus,

$$
\max \left\{\mu\left(A_{n} \cup A\right)-\mu(A), \mu\left(A_{n} \cup A\right)-\mu\left(A_{n}\right)\right\} \rightarrow 0,
$$

i.e., the sequence $\left\{A_{n}\right\}_{n=1}^{\infty}$ tends to the set $A$ with respect to the metric $D_{\mu}$.

On the other hand assume $\left\{A_{n}\right\}_{n=1}^{\infty}$ tends to $A$ with respect to the metric $D_{\mu}$, i.e.,

(4) $\mu\left(A_{n} \cup A\right)-\mu(A) \rightarrow 0$ and

(5) $\mu\left(A_{n} \cup A\right)-\mu\left(A_{n}\right) \rightarrow 0$. 
We show that

(6) $\operatorname{Lim}\left(A_{n} \cup A\right)=A$.

Assume, on the contrary, that there is a subsequence $\left\{A_{n_{i}}\right\}_{i=1}^{\infty}$ with $\operatorname{Lim}\left(A_{n_{i}} \cup A\right)$ $=B \neq A$. Then $A \subset B$ and (2) imply $\mu(A)<\mu(B)$, a contradiction to (4).

Note that (6) implies

(7) $\operatorname{Ls} A_{n} \subset A$.

Now suppose there exists a subsequence $\left\{A_{n_{j}}\right\}_{j=1}^{\infty}$ with $\operatorname{Lim} A_{n_{j}}=C \neq A$. By (7) we have $C \subset A$ and, therefore, by (2), $\mu(C)<\mu(A)$. Then (6) implies a contradiction to (5). So we have proved $\operatorname{Lim} A_{n}=A$, i.e., $\left\{A_{n}\right\}_{n=1}^{\infty}$ tends to $A$ with respect to the Hausdorff distance.

Now we show some facts concerning the metric $D_{\mu}$. Some of them are obvious and their proofs are omitted.

Let $X$ be a fixed continuum and let $\mu$ be a Whitney map satisfying (3).

FACT 5. Consider $2^{X}$ as a metric space with the metric $D_{\mu}$, and let $\mathscr{A} \subset 2^{X}$ be an ordered arc. Then $\left.\mu\right|_{\mathscr{A}}: \mathscr{A} \rightarrow[0, \infty)$ is an isometry.

FACT 6. Let $x \in A \in 2^{X}$. Then $D_{\mu}(A,\{x\})=\mu(A)$. In other words, the distance between $a$ set and any point in the set does not depend on the choice of the point.

FACT 7. Let $\mathscr{A}$ be an ordered arc contained in $2^{X}$ and let $P \in 2^{X}$. Denote by $A_{0}$ either the only set in $\mathscr{A}$ satisfying $\mu\left(A_{0}\right)=\mu(P)$ if such a set does exist, or $\cap \mathscr{A}$ if $\mu(P)<\mu(A)$ for each $A \in \mathscr{A}$, or $\cup \mathscr{A}$ if $\mu(P)>\mu(A)$ for each $A \in \mathscr{A}$. Then $\inf \left\{D_{\mu}(A, P): A \in \mathscr{A}\right\}=D_{\mu}\left(A_{0}, P\right)$.

Proof. Take a set $A \in \mathscr{A}$. We have to show $D_{\mu}\left(A_{0}, P\right) \leqslant D_{\mu}(A, P)$. Consider two cases:

Case 1. $A_{0} \subset A$. Then

$$
D_{\mu}(A, P)=\mu(A \cup P)-\mu(P) \geqslant \mu\left(A_{0} \cup P\right)-\mu(P)=D_{\mu}\left(A_{0}, P\right) .
$$

Case 2. $A \subset A_{0}$. Then by (3) we have

$$
D_{\mu}(A, P)=\mu(A \cup P)-\mu(A) \geqslant \mu\left(A_{0} \cup P\right)-\mu\left(A_{0}\right)=D_{\mu}\left(A_{0}, P\right) .
$$

This completes the proof.

FACT 8. Let $D$ be any metric on $2^{X}$ equivalent to the Hausdorff metric. Then the continuity of a Whitney map $\mu$ means

$$
\forall \varepsilon>0 \exists \delta>0 \forall A, B \in 2^{X}: D(A, B)<\delta \Rightarrow|\mu(A)-\mu(B)|<\varepsilon .
$$

If we replace $D$ by $D_{\mu}$ we can put $\delta=\varepsilon$.

Proof. We have to show $D_{\mu}(A, B)<\varepsilon$ implies $|\mu(A)-\mu(B)|<\varepsilon$. Assume $\mu(A)$ $\geqslant \mu(B)$. Then

$$
\varepsilon>D_{\mu}(A, B)=\mu(A \cup B)-\mu(B) \geqslant \mu(A)-\mu(B),
$$

and we are done.

To end the paper we ask some questions connected with condition (3). We say that two Whitney maps $\mu_{1}$ and $\mu_{2}$ are equivalent if for every $t$ there exist $t^{\prime}$ and $t^{\prime \prime}$ such that $\mu_{1}^{-1}(t)$ is homeomorphic to $\mu_{2}^{-1}\left(t^{\prime}\right)$ and $\mu_{2}^{-1}(t)$ is homeomorphic to $\mu_{1}^{-1}\left(t^{\prime \prime}\right)$. 
Question 9. Given any Whitney map $\mu_{1}$ is there a Whitney map $\mu_{2}$ which is equivalent to $\mu_{1}$ and satisfies (3)?

Question 10. Given any continuum $X$ and any Whitney map $\mu: 2^{X} \rightarrow[0, \mu(X)]$ does there exist a homeomorphism $h$ from $[0, \mu(X)]$ into $[0, \infty)$ such that $h \circ \mu$ is a Whitney map satisfying (3)?

\section{REFERENCES}

1. Sam B. Nadler, Jr., Hyperspaces of sets, Dekker, New York, 1978.

2. L. E. Ward, Jr., Extending Whitney maps, Pacific J. Math. 93 (1981), 465-469.

Ul. Beniowskiego 16 M. 2, 53 - 210 Wroclaw, Poland 